\title{
Genetic variation for an aphid wing polyphenism is genetically linked to a naturally occurring wing polymorphism
}

\author{
Christian Braendle ${ }^{1,2, *, \dagger}$, Ilvy Friebe ${ }^{2}$, Marina C. Caillaud $^{3}$ and David L. Stern ${ }^{2}$ \\ ${ }^{1}$ Laboratory for Development and Evolution, Department of Zoology, University Museum of Zoology, \\ Downing Street, Cambridge CB2 3EF, UK \\ ${ }^{2}$ Department of Ecology E Evolutionary Biology, Princeton University, Princeton, NF 08544, USA \\ ${ }^{3}$ Department of Biology, Ithaca College, Ithaca, NY 14850, USA
}

\begin{abstract}
Many polyphenisms are examples of adaptive phenotypic plasticity where a single genotype produces distinct phenotypes in response to environmental cues. Such alternative phenotypes occur as winged and wingless parthenogenetic females in the pea aphid (Acyrthosiphon pisum). However, the proportion of winged females produced in response to a given environmental cue varies between clonal genotypes. Winged and wingless phenotypes also occur in males of the sexual generation. In contrast to parthenogenetic females, wing production in males is environmentally insensitive and controlled by the sex-linked, biallelic locus, aphicarus (api). Hence, environmental or genetic cues induce development of winged and wingless phenotypes at different stages of the pea aphid life cycle. We have tested whether allelic variation at the api locus explains genetic variation in the propensity to produce winged females. We assayed clones from an $\mathrm{F}_{2}$ cross that were heterozygous or homozygous for alternative api alleles for their propensity to produce winged offspring. We found that clones with different api genotypes differed in their propensity to produce winged offspring. The results indicate genetic linkage of factors controlling the female wing polyphenism and male wing polymorphism. This finding is consistent with the hypothesis that genotype by environment interaction at the api locus explains genetic variation in the environmentally cued wing polyphenism.
\end{abstract}

Keywords: Acyrthosiphon pisum; alternative phenotypes; aphicarus; phenotypic plasticity; polyphenism; polymorphism

\section{INTRODUCTION}

All organisms use environmental cues to alter genetic programmes that generate variable phenotypic responses (Nijhout 1999; Stearns 1989; West-Eberhard 1989, 2003). These gene-environment interactions allow organisms to display phenotypic plasticity to cope with environments that are variable but have some predictability. In multi-cellular organisms, phenotypic plasticity can result from subtle or profound changes in developmental programmes. When discrete alternative phenotypes result from gene-environment interactions, such as the alternative castes in a social insect colony, they are called polyphenisms. Despite the ubiquity of plasticity and its importance in mediating how organisms interact with their environment, the mechanisms regulating alternative phenotypes are poorly understood.

Environmentally induced plasticity and genetic variation can produce similar phenotypic variation (Nijhout 1999). For example, variation in body size is determined both by genetic variation, often distributed among multiple loci, and by an interaction of genotype and environment (Partridge et al. 1994). This may indicate

\footnotetext{
*Author for correspondence (braendle@ijm.jussieu.fr).

${ }^{\dagger}$ Present address: Institut Jacques Monod, CNRS, Universités 6 et 7, Tour 43, 2 place Jussieu, 75251 Paris Cedex 05, France.
}

that phenotypic plasticity and natural genetic variation in a trait are mediated by the same genes. Alternatively, novel mechanisms may have evolved to allow flexible phenotypic responses to the environment. At the moment, there are few data with which to test these alternatives directly. However, there are data on mutations observed in the laboratory that may be relevant to this problem.

For example, in the buckeye butterfly, Precis coenia, the autumn morph is usually induced by low temperature and short days, but if individuals are homozygous for the recessive allele of the rosa gene (a spontaneous laboratory mutation), they produce the autumn phenotype independent of environmental conditions (Rountree \& Nijhout 1995).

Another example is the relationship between phenocopies, which are environmentally induced phenotypic changes that mimic effects of mutations, and genocopies, which are mutationally induced phenotypic changes that mimic environmentally induced changes. Phenocopies have been most thoroughly studied in laboratory populations of Drosophila, where phenocopies have been induced to mimic a wide variety of mutations. In a series of classic experiments, Waddington (1956) demonstrated that the frequency of phenocopies can be increased with selection. This indicates that genetic variation exists for susceptibility to phenocopies, which may be analogous 
to the genetic variation that exists in natural populations for the susceptibility of polyphenism induction. Gibson \& Hogness (1996) demonstrated that some of the genetic variation for susceptibility to ether-induced bithorax phenocopies resides at the $U b x$ locus, the gene at which bithorax mutants can be generated. In cases where polyphenisms mimic phenotypes generated by a polymorphism, one specific question is whether genotype by environment interaction for polyphenism induction occurs at the same loci that control the polymorphism. Answering this question would also help to clarify the evolutionary relationship between genetic and environmental control of such adaptive phenotypic variation.

We have started to address this question by examining the environmental and genetic induction of winged and wingless phenotypes in the pea aphid, Acyrthosiphon pisum (Hemiptera: Aphididae). During the parthenogenetic generations of the life cycle (see Electronic Appendix; figure 1), pea aphid females develop without wings under favourable environmental conditions, but when the host-plant quality declines, or the plant becomes overcrowded, females produce offspring that develop wings (wing polyphenism) and then may disperse by flight (Müller et al. 2001). These cues act via the mother and affect the phenotype of developing embryos shortly before birth (Sutherland 1969a,b). The winged female is typically fully winged, flight-capable and exhibits a variety of characteristics associated with flight, including (but not limited to) fully developed thoracic wing musculature, heavy sclerotization of head and thorax, ocelli and specialized antennae. The wingless female produces no wings or wing rudiments, no ocelli and smaller compound eyes (Kalmus 1945; Kring 1977; Kawada 1987; Tsuji \& Kawada 1987). The winged phenotype further differs from the wingless phenotypes by showing a longer developmental time, longer reproductive period, lower offspring production and a prolonged longevity (e.g. MacKay \& Wellington 1975).

In the single sexual generation of the pea aphid life cycle, females are always wingless and the males are either winged or wingless (Eastop 1971; Blackman \& Eastop 2000). The production of different male phenotypes is insensitive to environmental variation and controlled by the X-linked, biallelic locus aphicarus (api) (Smith \& MacKay 1989; Caillaud et al. 2002; Braendle et al. in press). The alternative alleles at the api locus cause the winged $\left(a p i^{\mathrm{w}}\right)$ or wingless $\left(a p i^{\mathrm{wl}}\right)$ male phenotype (wing polymorphism). (Males are haploid for the $\mathrm{X}$ chromosome owing to the XX:XO sex determination system (Blackman 1987). Therefore, depending on its api genotype, an aphid clone-consisting of genetically identical parthenogenetic females that are diploid for the $\mathrm{X}$ chromosome-will produce only winged males $\left(a p i^{\mathrm{w}} / a p i^{\mathrm{w}}\right)$, only wingless males $\left(a p i^{\mathrm{wl}} / a p i^{\mathrm{wl}}\right)$, or both male phenotypes in equal proportions $\left(a p i^{\mathrm{w}} / a p i^{\mathrm{wl}}\right)$ (Caillaud et al. 2002; Braendle et al. in press).

The coexistence of a wing polyphenism and a wing polymorphism in natural populations of the pea aphid provides an ideal system for examining the mechanistic relationship between genetic and environmental induction of alternative phenotypes. Pea aphid clones differ considerably in their propensity to produce winged offspring in response to a given environmental cue, indicating the presence of genotype by environment interactions for this trait (Markkula 1963; Lowe \& Taylor 1964; Sutherland 1969a,b, 1970; MacKay \& Lamb 1979; Lamb \& MacKay 1983; Weisser \& Braendle 2001). One hypothesis is that genetic variation in the propensity to produce winged parthenogenetic females is due to genotype by environment interaction at the api locus. In an initial test of this hypothesis, we examined whether clones with different api genotypes vary in their propensity to produce winged parthenogenetic females. In two assays, we measured variation in the propensity to produce winged females in $\mathrm{F}_{2}$ clones segregating for the three different api genotypes. First, we measured the propensity of $\mathrm{F}_{2}$ clones to produce winged offspring in response to a wing-inducing cue, a combined crowding and starvation stimulus. Second, to more closely mimic natural conditions, we measured the production of winged offspring by $\mathrm{F}_{2}$ clones at a constant (winginducing) density over 12 consecutive parthenogenetic generations. The results of both experiments suggest genetic linkage of factors controlling the wing polyphenism and wing polymorphism. This outcome is consistent with the hypothesis that genotype by environment interaction at the api locus explains genetic variation in the inducibility of the polyphenism.

\section{MATERIALS AND METHODS}

\section{(a) Aphid rearing and handling}

Parthenogenetic aphid cultures were maintained under longday conditions (L:D 16:8) at $20^{\circ} \mathrm{C}$. Aphids were reared in plastic Petri dishes (diameter: $55 \mathrm{~mm}$; Fisher) with a single leaf of Medicago arborea inserted into $3 \mathrm{ml}$ of $2 \%$ agar (Fisher) containing $1 \mathrm{~g} \mathrm{~L}^{-1}$ plant fertilizer (Miracle-Gro). Circular filter papers (diameter: $55 \mathrm{~mm}$, Whatman) were added to the dishes to absorb condensation. In stock cultures, aphids were transferred to fresh leaves every 10-15 days. In all experiments, leaves of $M$. arborea were collected from several plants, usually of the same age and were distributed at random among experimental replicates to avoid potential confounding effects owing to differences in plant material. In the experiments, aphids were handled and transferred to fresh leaves using a fine paintbrush.

\section{(b) Experiment 1: clonal variation in wing morph production in response to a crowding/starvation stimulus \\ (i) Aphid clones}

The clones used in this experiment were derived from an $\mathrm{F}_{2}$ mapping population segregating for api (Braendle et al. in press). Two parental clones homozygous for alternative $a p i$ alleles (PBR8 $\left(a p i^{\mathrm{w}} / a p i^{\mathrm{w}}\right)$ and LSR1 $\left(a p i^{\mathrm{wl}} / a p i^{\mathrm{wl}}\right)$, collected in 1998, from Medicago sativa in Tompkins county, NY, USA) were crossed to generate $\mathrm{F}_{1}$ clones. Two $\mathrm{F}_{1}$ clones $\left(a p i^{\mathrm{w}} /\right.$ $a p i^{\mathrm{wl}}$ ) resulting from reciprocal parental crosses were crossed to each other to generate $\mathrm{F}_{2}$ clones with the three possible api genotypes: $\left(a p i^{\mathrm{w}} / a p i^{\mathrm{w}}\right),\left(a p i^{\mathrm{w}} / a p i^{\mathrm{wl}}\right)$ and $\left(a p i^{\mathrm{wl}} / a p i^{\mathrm{wl}}\right)$. For this experiment, we used a total of $21 \mathrm{~F}_{2}$ clones: $5 a p i^{\mathrm{w}} / a p i^{\mathrm{w}}$, $8 a p i^{\mathrm{w}} / a p i^{\mathrm{wl}}$ and $8 a p i^{\mathrm{wl}} / a p i^{\mathrm{wl}}$ clones. The api genotype of all clones was determined as described in Braendle et al. (in press) and carried out prior to the experiment. 


\section{(ii) Experimental design}

We measured the clonal propensity to produce winged phenotypes (morphs) by exposing adult females to a combined crowding/starvation stimulus-conditions known to trigger an increased production of wing morphs in the pea aphid (Lamb \& MacKay 1983; Weisser \& Braendle 2001). The production of wing morphs is influenced by the maternal phenotype: winged females produce no or few winged offspring (Lees 1966; Sutherland 1969b, 1970; MacKay \& Wellington 1975). We therefore attempted to minimize such maternal effects by maintaining clones for three generations prior to the experimental treatment in low-density cultures produced by wingless mothers (see Electronic Appendix, figure 2). We selected five adult wingless females ( $\mathrm{G} 1=$ generation 1 ) per clone from low-density stock cultures and transferred each to a separate dish. These G1 individuals were allowed to reproduce for 2-3 days, after which we selected one replicate containing at least 10 offspring (G2; the other four replicates were discarded). Ten offspring per clone were transferred to separate Petri dishes. After these nymphs had matured into adults we selected five G2 wingless females and transferred each to a separate dish (discarding the remaining five individuals). After 2-3 days, we collected seven offspring (G3) from each female ( $n=35$ per clone) and transferred each to a separate dish (any additional offspring were discarded). Approximately 3 days after maturity, 20 wingless G3 females were selected for use in the experiment (the other 15 G3 females were discarded).

The 20 experimental aphids per clone were randomly allocated to either a treatment $(n=10)$ or a control group $(n=10)$. The 10 aphids in the treatment group were placed together in a single small Petri dish (diameter $39 \mathrm{~mm}$ ) without a leaf and control animals were kept singly in slightly larger Petri dishes (diameter $55 \mathrm{~mm}$ ) containing a leaf of $M$. arborea inserted into agar. Pieces of moist filter paper were added to Petri dishes of the treatment group to prevent desiccation. After $24 \mathrm{~h}$, each adult female from treatment and control groups was transferred to a separate Petri dish. We collected the offspring produced by each female every $24 \mathrm{~h}$ over the next $72 \mathrm{~h}$. Previous studies have shown that after exposure to a wing-inducing stimulus of about $24 \mathrm{~h}$, an increased production of winged offspring is observed for several days, ranging from 1 to 5 days (Sutherland 1969a; Mackay \& Wellington 1977; Weisser \& Braendle 2001). Offspring were phenotyped for wing absence versus presence at maturity.

The experiment was carried out at two different times (separated by four months), each time using a different set of $\mathrm{F}_{2}$ clones.

\section{(iii) Statistical analysis}

We estimated the clonal propensity for wing morph production using two measures. First, we measured the proportion of aphids $(n=10$ for both treatment and control group per clone) that produced at least one winged offspring. Second, we measured the proportion of winged individuals among an aphid's offspring (using the mean proportion of winged offspring produced per clone for statistical tests). Replicates in which the experimental aphids produced fewer than five offspring or no offspring at all (e.g. owing to mortality) were excluded from the analysis. The data were analysed using ANOVA (GLM; JMP, v. 4.0), testing for the fixed effects of date of experiment (to test for differences between the two different time points at which the experiment was carried out), treatment (crowding/starvation versus control group) and api genotype (nested in date of experiment). We also tested for the same effects to detect potential differences in the number of replicates sampled and the number of total offspring produced. In separate ANOVAs, we further tested for clonal differences in the proportion of winged offspring produced in response to the starvation/ crowding stimulus within each api genotype, testing for the effects of clone (nested in date of experiment) and date of experiment. To satisfy assumptions of homogeneity of variance and normality of error, the data were transformed where necessary (Sokal \& Rohlf 1995).

\section{(c) Experiment 2: clonal variation in wing morph production at constant density}

\section{(i) Aphid clones}

We used a total of 23 clones derived from the same $\mathrm{F}_{2}$ population (Braendle et al. in press) as in the first experiment: $8 a p i^{\mathrm{w}} / a p i^{\mathrm{w}}, 7 a p i^{\mathrm{w}} / a p i^{\mathrm{wl}}$ and $8 a p i^{\mathrm{wl}} / a p i^{\mathrm{wl}}$ clones.

\section{(ii) Experimental design}

Measuring the clonal propensity to produce winged offspring is complicated by the fact that a variable array of internal or external factors affects morph induction and may confound these measurements. Previous studies have shown that a single aphid clone may vary dramatically in its propensity to produce winged offspring over time (e.g. Blackman 1979). It is unclear to what extent variable environmental factors, such as host plant quality, or internal factors (e.g. maternal and grand-maternal effects) contribute to the observed variation. In any case, it suggests that assessing wing morph production of a clone at a single time point may be unreliable, despite carrying out carefully controlled and standardized experiments. For example, it is probable that host plant variation (which is difficult, if not impossible to standardize or measure) may affect such an experiment. We therefore aimed to validate the results of experiment 1 by repeatedly screening $\mathrm{F}_{2}$ clones with different api genotypes for their production of winged offspring (see Electronic Appendix, figure 3). In contrast to the first experiment, aphids were not exposed to a starvation/crowding stimulus but were kept at a constant density and the proportion of winged offspring produced by a clone was measured in each generation. For each clone, we established four dishes each containing eight individuals $(n=32$ per clone). This density was chosen to allow newly born aphids to develop on a single leaf for approximately 10-15 days, during which time aphids had fully matured and had started to reproduce for about 3-5 days. (In pilot tests, we confirmed that this experimental procedure resulted in wing morph production among the $F_{2}$ clones.) In each generation, we determined the phenotype (winged or wingless) of the adult females. From each clone, we then selected the dish containing the most offspring (usually around 50-100 offspring). From this dish, we selected 32 of the youngest offspring (first- and secondinstar nymphs) and distributed them equally among four dishes with fresh leaves. Whenever a dish contained fewer than 32 offspring, all of the offspring in the dish were distributed equally among the four dishes. The selection of dishes with the highest densities ensured that the derived offspring were produced mainly by wingless females, as winged females have a prolonged nymphal developmental 
time and pre-reproductive period when adult (MacKay \& Wellington 1975; Campbell \& Mackauer 1977; Tsumuki et al. 1990). This resulted in a lower contribution of winged females to the total number of offspring relative to wingless females, thereby avoiding the problem of selecting a large proportion of offspring descending from winged females, which are predominantly wingless and mainly produce wingless offspring themselves (Lees 1966; Sutherland 1969b, 1970; MacKay \& Wellington 1975). Moreover, our experimental procedure selected offspring born by young mothers of similar age. As females of the pea aphid have been reported to produce more winged offspring at an early age (Mackay \& Wellington 1977), this may have been one of the reasons for the observed production of winged offspring in the experimental progeny despite the absence of a direct experimental stimulus. In addition, our statistical analysis (see $\$ 2 \mathrm{c}$ (iii)) controls for differences in density and the number of winged mothers present. We measured the proportion of winged offspring produced in 14 consecutive generations. The two initial generations were excluded from analysis to avoid potential confounding measurements owing to maternal and grand-maternal effects stemming from unequal rearing conditions in the stock cultures.

\section{(iii) Statistical analysis}

For each generation, we estimated the clonal propensity to produce winged offspring by measuring the percentage of winged adult females $(n=32$, barring losses owing to mortality). When a percentage value was based on a number of individuals smaller than 10 (e.g. owing to mortality), the data point was excluded from the analysis. We used a mixed-model ANCOVA (REML procedure, JMP v. 4.0) to test for the fixed effects of generation and api genotype and the random effect of clone (nested in api genotype). In addition, the analysis controlled for the effects of density and the proportion of winged mothers (both effects treated as covariates). Density was measured as the number of live adult females present at the time of transfer, and the percentage of winged females among these live adult females was the proportion of winged mothers. To satisfy assumptions of homogeneity of variance and normality of error, the data were transformed where necessary (Sokal \& Rohlf 1995).

\section{RESULTS}

\section{(a) Experiment 1: clonal variation in wing morph production in response to a crowding/starvation stimulus}

The experimental stimulus of crowding and starvation resulted in an increased production of winged offspring relative to the control group, and clones with different $a p i$ genotypes showed significant differences in wing morph production (table 1). Clones homozygous for $a p i^{\mathrm{w}}$ exhibited a significantly lower propensity to produce winged female offspring compared with clones homozygous for $a p i^{\text {wl }}$ and $a p i$ heterozygotes (Tukey's HSD, $\alpha=0.05)$. The latter two genotypes did not differ in their production of winged offspring (Tukey's HSD, $\alpha=0.05$; table 1, figure $1 a$ ). The same effect of api genotype on wing morph production was detected when testing for differences in the mean percentage of winged female offspring produced (table 2; figure $1 b$ ). Clones homozygous for $a p i^{\mathrm{w}}$ showed a lower propensity to produce winged female offspring relative to clones with alternative api genotypes (Tukey's HSD; $\alpha=0.05)$. The $a p i^{\mathrm{w}} / a p i^{\mathrm{w}}$ clones showed little variation in the proportion of winged females produced in the treatment group (mean percentage range $0-3 \%)$, while $a p i^{\mathrm{w}} / a p i^{\mathrm{wl}}(0-27 \%)$ and $a p i^{\mathrm{wl}} / a p i^{\mathrm{wl}}$ clones (3-51\%) exhibited considerable clonal variation in wing morph production (figure 2). Separate analyses (ANOVAs) on the proportion of winged offspring produced within each api genotype (treatment group only) show that there was significant clonal variation in wing morph production within the two genotypes $a p i^{\mathrm{w}} / a p i^{\mathrm{wl}}$ (effect of clone(date experiment): $F_{6,55}=3.00, p=0.0132$ ) and $a p i^{\mathrm{wl}} / a p i^{\mathrm{wl}}$ (clone (date exp.): $F_{6,57}=4.09, p=0.0018$ ) but not within the genotype $a p i^{\mathrm{w}} / a p i^{\mathrm{w}}$ (effect of clone(date experiment): $\left.F_{3,34}=0.52, p=0.67\right)$.

The mean number of offspring produced per clone during the $72 \mathrm{~h}$ after the $24 \mathrm{~h}$ treatment/control period differed significantly between treatment and control group (ANOVA: $F_{1,35}=6.25, p=0.02$ ), with individuals that were exposed to the crowding/starvation stimulus producing more offspring than control individuals. However, offspring production did not differ between experimental dates (ANOVA: $F_{1,35}=1.90, p=0.18$ ) or different $a p i$ genotypes (ANOVA: $F_{4,35}=2.0, p=0.12$ ). Furthermore, there was no difference in the mean number of clonal replicates sampled between different api genotypes (ANOVA: $F_{4,35}=1.93, p=0.13$ ). Thus, differences in offspring production or replicates sampled did not account for the observed differences in wing morph production between different api genotypes.

Very few aphids in the crowding/starvation treatment produced any offspring during the $24 \mathrm{~h}$ during which the stimulus was applied (the $>2000$ experimental aphids produced a total of six offspring). Individuals of the control group produced an average of $2.54 \pm 0.23$ individuals during this period, of which $5.00 \pm 1.63 \%$ were winged. For control animals, there was no evident effect of wing morph production during this period on the subsequent $72 \mathrm{~h}$ of wing morph production (data not shown).

\section{(b) Experiment 2: clonal variation in wing morph production at constant density}

Given the significant effect of api genotype in the previous experiment, we sought to validate these results with a prolonged experimental treatment that imitated natural conditions more closely. We therefore assayed wing morph production in cultures at a wing-inducing density over 12 consecutive generations. We found that although wing morph production was highly variable across different generations, the factors api genotype, clone and density showed significant effects on wing morph production (table 3; figure 3). Clones with different api genotypes differed in their production of winged offspring as in experiment 1; clones homozygous for $a p i^{\mathrm{w}}$ produced fewer winged offspring than $a p i^{\mathrm{w}} / a p i^{\mathrm{wl}}$ and $a p i^{\mathrm{wl} /} / a p i^{\mathrm{wl}}$ clones, while the latter two api genotypes did not differ in wing morph production (Tukey's HSD, $\alpha=0.05$; figure 3). The significant effect of the factor clone shows that there is variation in wing morph production between clones within each api genotype (table 3). Similar to the first experiment, clones homozygous for $a p i^{\mathrm{w}}$ exhibit less variation in parthenogenetic female wing production than the other api genotypes (figure 3). 
(a)

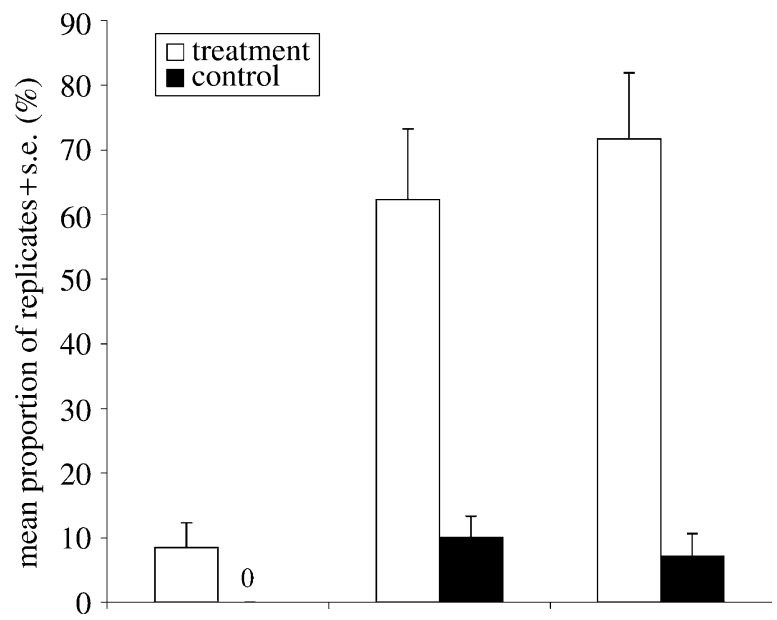

(b)

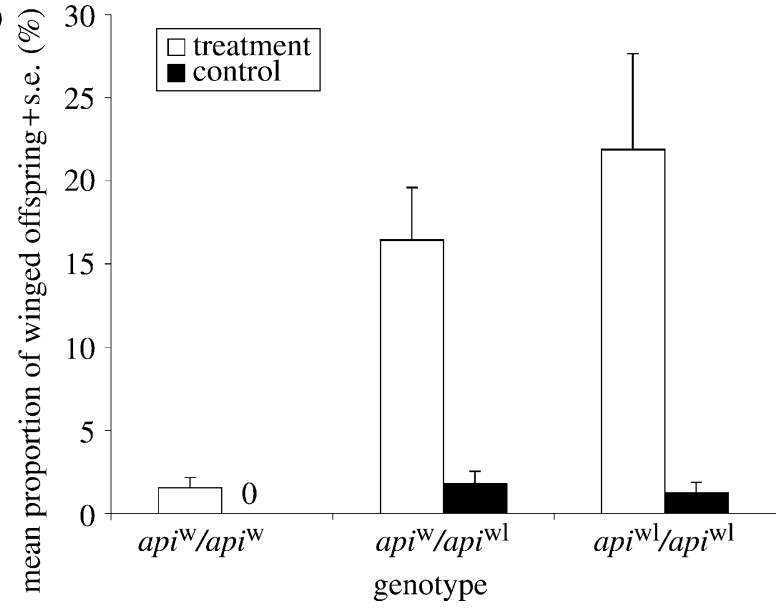

Figure 1. Experiment 1: Differences in the propensity to produce winged offspring among $\mathrm{F}_{2}$ clones with different api genotypes in response to a starvation/crowding stimulus. (a) The propensity to produce winged offspring is measured as the proportion of clonal replicates containing at least one winged offspring. (b) The propensity to produce winged offspring is measured as the mean percentage of winged offspring produced. (Note that control individuals of the genotype $a p i^{\mathrm{w}} / a p i^{\mathrm{w}}$ produced zero winged offspring.)
Table 1. Test of effect of crowding treatment and api genotype on proportion of replicates producing winged offspring. (Analysis of variance testing for the fixed effects of date of experiment, treatment and api genotype (nested in date of experiment) on wing morph production, measured as the clonal proportion of replicates containing at least one winged offspring produced within $72 \mathrm{~h}$ after the treatment (arcsine transformed data). Interactions terms were non-significant and therefore removed from the model.)

\begin{tabular}{lrrrr}
\hline source & d.f. & SS & \multicolumn{1}{c}{$F$} & \multicolumn{2}{c}{$p$} \\
\hline date of experiment & 1 & 0.2709 & 2.7427 & 0.1066 \\
treatment & 1 & 4.5329 & 45.9000 & $<0.0001$ \\
api genotype (date exp.) & 4 & 1.4425 & 3.6517 & 0.0137 \\
error & 35 & 3.4565 & & \\
\hline
\end{tabular}

\section{DISCUSSION}

The two alternative alleles at the api locus cause the winged and wingless male phenotypes in the sexual phase of the pea aphid life cycle (Smith \& MacKay 1989; Caillaud et al. 2002; Braendle et al. in press). In this study we found that parthenogenetic females with different api genotypes differ in their propensity to produce winged versus wingless parthenogenetic females in response to environmental cues. In the first experiment, we imposed stringent control over the environmental cues and found a strong association of api genotype and production of winged parthenogenetic females (figure 1; tables 1 and 2). There was also a weak effect of the experimental date on the percentage of winged offspring produced (table 2). In the second experiment, we aimed to confirm and extend the results of the first experiment by rearing aphids in more natural conditions and assaying wing production over 12 generations. We again found a significant association of genotype and female wing production that persisted over time. In addition, uncontrolled factors imposed a strong trend on wing production by all clones irrespective of api genotype (figure 3). It is probable that this temporal variation in wing morph production resulted from variation in the plant material used for feeding the

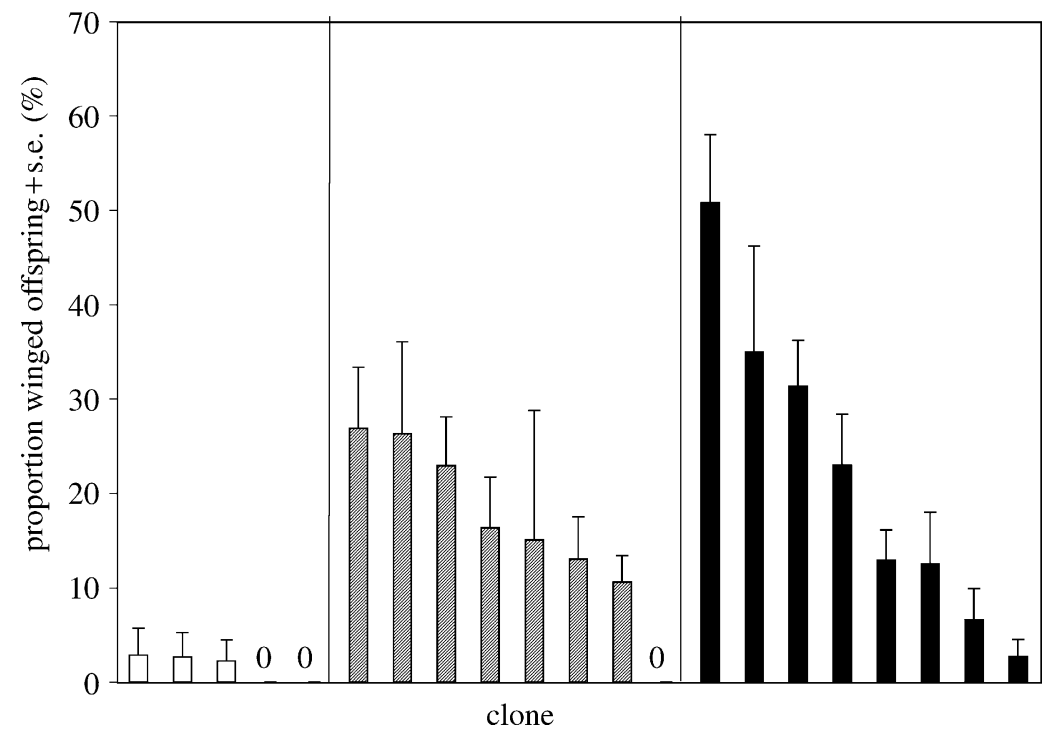

Figure 2. Experiment 1: Among-clone variation in wing morph production in response to starvation/crowding stimulus within different api genotypes (percentage data for treatment group only). For each api genotype, clonal proportions are rank-ordered. 
Table 2. Test of effect of crowding treatment and api genotype on proportion of winged offspring produced. (Analysis of variance testing for the fixed effects of date of experiment, treatment and api genotype (nested in date of experiment) on wing morph production (measured as the clonal mean proportion of winged offspring produced within $72 \mathrm{~h}$ after the treatment (arcsine transformed data). Interactions terms were non-significant and therefore removed from the model.)

\begin{tabular}{lrlrr}
\hline source & d.f. & SS & \multicolumn{2}{c}{$F$} \\
\hline date of experiment & 1 & 0.0780 & 4.3012 & 0.0455 \\
treatment & 1 & 0.8276 & 45.6142 & $<0.0001$ \\
api genotype (date exp.) & 4 & 0.2281 & 3.1431 & 0.0262 \\
error & 35 & 0.6350 & & \\
\hline
\end{tabular}

aphids. This factor may also explain the effect of experimental date on winged offspring production in the first experiment.

In both experiments, $\mathrm{F}_{2}$ clones homozygous for $a p i^{\mathrm{wl}}$ and heterozygous clones $\left(a p i^{\mathrm{w}} / a p i^{\mathrm{wl}}\right)$ produced a similar and significantly higher proportion of winged females than did clones homozygous for the $a p i^{\text {w }}$ allele (figures 1-3). This result implies that genetic variation in the propensity to produce winged females is genetically linked to api and that $a p i^{\mathrm{wl}}$ chromosomes carry a factor dominant to factors on the $a p i^{\mathrm{w}}$ chromosome for the propensity to produce the winged phenotype in females. We do not know the extent of linkage between api and the factor(s) contributing to variation in the polyphenism. In the extreme, the linkage could reflect pleiotropic effects of api. We can resolve this question only by (i) assaying a larger sample of $\mathrm{F}_{2}$ clones or clones from natural populations that segregate for $a p i$, (ii) cloning api and (iii) testing whether it functions to influence the polyphenism.

The gene(s) that affect the polyphenism and segregate with api did not account for all of the genetic variation in
Table 3. Test of effect of api genotype on the production of winged offspring over 12 consecutive generations at high density. (Mixed-model analysis of covariance testing for the effects of generation, api genotype and clone (nested in api genotype) on wing morph production measured as the percentage of winged offspring produced by each clone (arcsine transformed data). In addition, we controlled for the effects of density and the proportion of winged mothers present in clonal replicates. Interactions terms were non-significant and therefore removed from the model.)

\begin{tabular}{lrrrr}
\hline source & d.f. & \multicolumn{1}{l}{ SS } & \multicolumn{1}{l}{$F$} \\
\hline generation & 11 & 5.6831 & 8.8019 & $<0.0001$ \\
api genotype & 2 & 0.7084 & 6.0342 & 0.0089 \\
clone (api genotype) & 20 & 2.2208 & 1.8918 & 0.0144 \\
density & 1 & 0.8858 & 15.0914 & 0.0001 \\
proportion winged & 1 & 0.0133 & 0.2270 & 0.6343 \\
mothers & & & & \\
error & 208 & 25.3017 & & \\
\hline
\end{tabular}

the propensity to produce winged offspring. Within each genotypic class of api, there was significant variation between clones in the propensity to produce winged females (figure 2; table 3), indicating that there is additional genetic variation segregating for the trait. Clones homozygous for $a p i^{\mathrm{w}}$ produced uniformly low levels of winged females, whereas clones of the other two genotypes displayed significant variation in wing morph production. This implies that the factor segregating for female wing production is epistatic to the additional genetic variation. This is precisely what would be expected if this factor acts in the same developmental pathway as the remaining genetic variation.

We found wing induction in polyphenic females and polymorphic males to be inversely related. Clones producing only winged males $\left(a p i^{\mathrm{w}} / a p i^{\mathrm{w}}\right)$ rarely produced parthenogenetic winged females in the two experiments.

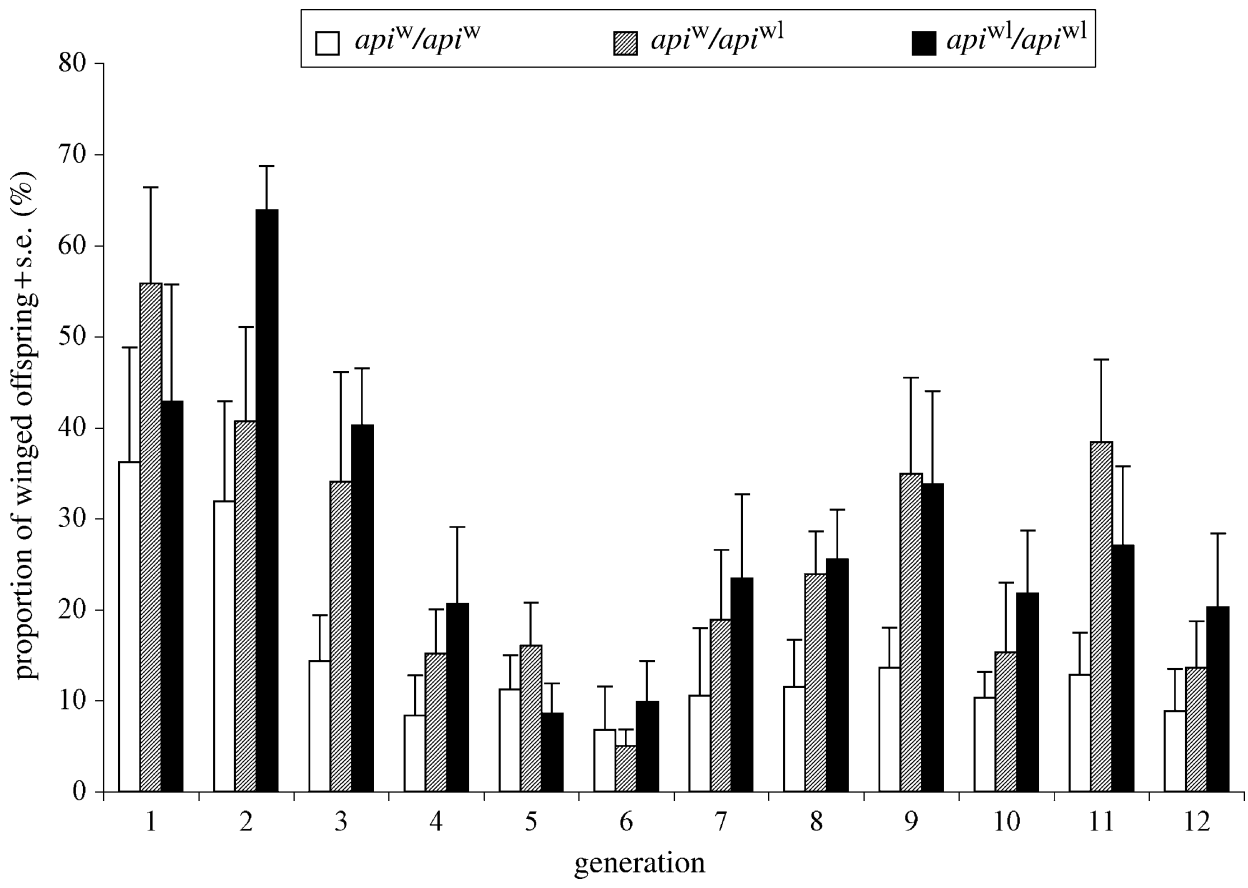

Figure 3. Experiment 2: Differences in the propensity to produce winged offspring among $\mathrm{F}_{2}$ clones with different api genotypes during 12 consecutive parthenogenetic generations (percentage data). White bars: genotype $a p i^{\mathrm{w}} / a p i^{\mathrm{w}}$; hatched bars: genotype $a p i^{\mathrm{w}} / a p i^{\mathrm{wl}}$; black bars: genotype $a p i^{\mathrm{wl}} / a p i^{\mathrm{wl}}$. 
The observed direction of the relationship could be due to chance selection of two parental lines segregating for these phenotypes in reversed phase, and further work is required to confirm the inverse linkage of the polyphenism and polymorphism, and to determine the ecological causes and consequences of this linkage. The direction of this relationship initially seemed counterintuitive, but it must be remembered that we have demonstrated only linkage between the traits, and not that they result from variation at the same gene. However, if they do reflect variation at the same gene, there is extensive precedent in the developmental biology literature for pleiotropic effects of single genes.

Polyphenisms are ideal systems to study gene-environment interactions that govern adaptive phenotypic plasticity. Yet it remains difficult to identify the key genes responsible for developmental divergence of alternative phenotypes because classical genetic analysis is impossible. Studying an organism in which both genetic and environmental induction mechanisms generate similar sets of alternative phenotypes provides an alternative experimental approach. First, the genes involved in the genetic induction mechanisms can be identified using genetics. Second, after identification, these genes can be tested for their potential roles in the environmental induction mechanism. In the example of the pea aphid, molecular and functional characterization of api could consequently contribute to a better understanding of the mechanisms controlling adaptive phenotypic plasticity and further may illuminate the evolutionary relationship between different control mechanisms underlying the development of alternative phenotypes.

We thank Greg Davis, Thomas Flatt, Laurent Keller, Alex Shingleton and two anonymous referees for critical and helpful comments on an earlier version of this manuscript. C.B. was supported by the Boehringer Ingelheim Fonds, the Roche Research Foundation, and the Janggen-PoehnStiftung. D.L.S. acknowledges NIH, the David \& Lucile Packard Foundation and Princeton University for financial support.

\section{REFERENCES}

Blackman, R. L. 1979 Stability and variation in aphid clonal lineages. Biol. F. Linn. Soc. 11, 259-277.

Blackman, R. L. 1987 Reproduction, cytogenetics and development. In Aphids: their biology, natural enemies $\mathcal{E}$ control, vol. 2A (ed. P. Harrewijn), pp. 163-195. Amsterdam: Elsevier.

Blackman, R. L. \& Eastop, V. F. 2000 Aphids on the World's crops: an identification and information guide. Chichester: Wiley.

Braendle, C., Caillaud, C. M., Stern, D. L. In press Genetic mapping of aphicarus - a sex-linked locus controlling a wing polymorphism in aphids Heredity.

Caillaud, C. M., Boutin, M., Braendle, C. \& Simon, J. C. 2002 A sex-linked locus controls wing polymorphism in males of the pea aphid, Acyrthosiphon pisum (Harris). Heredity 89, 346-352.

Campbell, A. \& Mackauer, M. 1977 Reproduction and population growth of the pea aphid (Homoptera: Aphididae) under laboratory and field conditions. Can. Entomol. 109, 277-284.

Eastop, V. F. 1971 Keys for the identification of Acyrthosiphon. Bull. Br. Mus. (Nat. Hist.) Entomol. 26, 1-115.
Gibson, G. \& Hogness, D. S. 1996 Effect of polymorphism in the Drosophila regulatory gene Ultrabithorax on homeotic stability. Science 271, 200-203.

Kalmus, H. 1945 Correlations between flight and vision, and particularly between wings and ocelli, in insects. Proc. $R$. Entomol. Soc. Lond. A20, 84-96.

Kawada, K. 1987 Forms and morphs of aphids. In Aphids, their biology, natural enemies and control, vol. 2A (ed. P. Harrewijn), pp. 255-266. Amsterdam: Elsevier.

Kring, J. B. 1977 Structure of the eyes of the pea aphid, Acyrthosiphon pisum. Ann. Entomol. Soc. Am. 70, 855-860.

Lamb, R. J. \& MacKay, P. A. 1983 Micro-evolution of the migratory tendency, photoperiodic response and developmental threshold of the pea aphid, Acyrthosiphon pisum. In Diapause and life cycle strategies in insects (ed. I. Hodek), pp. 209-217. The Hague: Dr W Junk Publishers.

Lees, A. D. 1966 The control of polymorphism in aphids. Adv. Insect Physiol. 3, 207-277.

Lowe, H. J. B. \& Taylor, L. R. 1964 Population parameters, wing production and behaviour in red and green Acyrthosiphon pisum (Harris) (Homoptera: Aphididae). Entomol. Exp. Appl. 7, 287-295.

MacKay, P. A. \& Lamb, R. J. 1979 Migratory tendency in aging populations of the pea aphid, Acyrthosiphon pisum. Oecologia 39, 301-308.

MacKay, P. A. \& Wellington, W. G. 1975 A comparison of the reproductive patterns of apterous and alate virginoparous Acyrthosiphon pisum (Homoptera: Aphididae). Can. Entomol. 107, 1161-1166.

Mackay, P. A. \& Wellington, W. G. 1977 Maternal age as a source of variation in the ability of an aphid to produce dispersing forms. Res. Popul. Ecol. 18, 195-209.

Markkula, M. 1963 Studies on the pea aphid, Acyrthosiphon pisum Harris (Hom., Aphididae), with special reference to the differences in the biology of the green and red forms. Ann. Agric. Fenn. 2, 1-30.

Müller, C. B., Williams, I. S. \& Hardie, J. 2001 The role of nutrition, crowding and interspecific interactions in the development of winged aphids. Ecol. Entomol. 26, 330-340.

Nijhout, H. F. 1999 Control mechanisms of polyphenic development. BioScience 49, 181-192.

Partridge, L., Barrie, B., Fowler, K. \& French, V. 1994 Evolution and development of body size and cell size in Drosophila melanogaster in response to temperature. Evolution 48, 1269-1276.

Rountree, D. B. \& Nijhout, H. F. 1995 Genetic control of a seasonal morph in Precis coenia (Lepidoptera, Nymphalidae). F. Insect Physiol. 41, 1141-1145.

Smith, M. A. H. \& MacKay, P. A. 1989 Genetic variation in male alary dimorphism in populations of pea aphid, Acyrthosiphon pisum. Entomol. Exp. Appl. 51, 125-132.

Sokal, R. F. \& Rohlf, F. J. 1995 Biometry. New York: W. H. Freeman.

Stearns, S. C. 1989 The evolutionary significance of phenotypic plasticity. BioScience 39, 436-445.

Sutherland, O. R. W. $1969 a$ The role of crowding in the production of winged forms by two strains of the pea aphid, Acyrthosiphon pisum. F. Insect Physiol. 15, 1385-1410.

Sutherland, O. R. W. $1969 b$ The role of the host plant in the production of winged forms by two strains of the pea aphid, Acyrthosiphon pisum. f. Insect Physiol. 15, 2179-2201.

Sutherland, O. R. W. 1970 An intrinsic factor influencing the alate production by two strains of the pea aphid, Acyrthosiphon pisum. F. Insect Physiol. 16, 1349-1354. 
Tsuji, H. \& Kawada, K. 1987 Development and degeneration of wing buds and indirect flight muscles in the pea aphid (Acyrthosiphon pisum (Harris)). Fpn. F. Appl. Entomol. Zool. 31, 247-252.

Tsumuki, H., Kawada, K., Nagatsuka, H. \& Kanehisa, K. 1990 Comparison of nutrient reservation in apterous and alate pea aphids, Acyrthosiphon pisum (Harris). 2. Aminoacid contents. Appl. Entomol. Zool. 25, 223-229.

Waddington, C. H. 1956 Genetic assimilation of the bithorax phenotype. Evolution 10, 1-13.

Weisser, W. W. \& Braendle, C. 2001 Body colour and genetic variation in winged morph production in the pea aphid. Entomol. Exp. Appl. 99, 217-223.
West-Eberhard, M. J. 1989 Phenotypic plasticity and the origins of diversity. Ann. Rev. Ecol. Syst. 20, 249-278.

West-Eberhard, M. J. 2003 Developmental plasticity and evolution. Oxford: Oxford University Press.

The supplementary Electronic Appendix is available at http://dx.doi. org/10.1098/rspb.2004.2995 or via http://www.journals.royalsoc.ac.uk.

As this paper exceeds the maximum length normally permitted, the authors have agreed to contribute to production costs. 